\title{
Biomatériaux et chirurgie orthopédique
}

La qualité des matériaux implantés en chirurgie orthopédique bénéficie des retombées de l'industrie aérospatiale. La prothèse idéale, résistante, biocompatible et capable d'une certaine plasticité reste cependant à développer.

\section{Pascal Christel \\ Chef de clinique - Assistant à l'hôpital Saint-Louis.}

\section{RÉFÉRENCES}

1. Sedel L, Christel P. Les prothèses articulaires. Solutions actuelles et projets d'avenir. Reo Biotechnol Méd 1981 ; 3 : 247-57.

2. Christel P, Sedel L. Les biomatériaux utilisés en chirurgie orthopédique : passé, présent et avenir. Reo Biotechnol Méd 1980 ; 2 : 137-41, 227-30.

3. Pharmaceutical manufacturers association guidelines for the assessment of drug and medical devices safety in animals. Washington DC, 1977.

4. Annual Book of ASTM Standards medica devices, section 13, vol 30, Philadelphia, 1983.

5. British Standards Institution. Evaluation of medical devices for biological hazards. BS 5736. Part 1, 1979, Parts 2, 3, 4, 1981, Part 5, 1982, Parts 6 and 7, 1983.

P. Christel : laboratoire de recherches orthopédiques, U.A. Cnrs 1161, 10, avenue de Verdun, 75010 Paris, et service de chirurgie orthopédique, hôpital Saint-Louis, 40, rue Bichat, 75475 Paris cedex 10
}

$\mathrm{m} / \mathrm{s} n^{\circ} 10$ vol. 2, dócembre 86 a chirurgie orthopédique connaît un essor considérable lié principalement au succès des prothèses articulaires. On pose actuellement en France 40000 à 50000 prothèses totales de hanche par an [1]. Ce développement important n'a pas été sans difficulté et de nombreux problèmes sont encore loin d'être résolus. Ceux-ci touchent à des domaines aussi divers que la science des matériaux (naturels et artificiels), la toxicologie, la biologie cellulaire, etc. Ainsi, la multidisciplinarité de la recherche est indispensable à l'élaboration de nouveaux matériaux à usage orthopédique [2]. Le but de cet article est de faire brièvement le point sur les connaissances actuellement acquises et sur les différentes voies de recherche à venir.

\section{Le cahier des charges}

Le squelette a essentiellement un rôle de soutien, donc de résistance mécanique. Les différents éléments qui le constituent, os, cartilage, ligaments, ont une structure tissulaire complexe, ayant des caractéristiques différentes selon les trois directions de l'espace. Les propriétés mécaniques de ces tissus dépendent de leur structure qui présente des renforcements à orientations préférentielles dans les zones d'hyper-sollicitations mécaniques. Les tissus du système squelettique allient résistance mécanique et élasticité. Ces caractéristiques ne sont pas figées ; il existe une adaptabilité (relation forme/fonction) par l'intermédiaire de processus de remodelage. La lubrification articulaire assure aux articulations un des plus bas coefficients de friction connu dans la nature et utilise des éléments (cartilage, liquide synovial) en perpétuel renouvellement. Ainsi, le remplacement par un matériau artificiel d'un ou de plusieurs des éléments du système squelettique ne peut-il être qu'approximatif, faisant appel à des matériaux homogènes (ayant les mêmes propriétés dans les trois directions de l'espace), inertes, sans capacité de renouvellement, donc sujets aux processus de dégradation physicochimique par vieillissement, fatigue ou usure.

\section{Les biomatériaux actuels}

Le marché mondial des implants orthopédiques représente un chiffre d'affaire annuel estimé à 2 milliards de dollars. C'est peu, comparé à d'autres secteurs biomédicaux, tel celui du médicament. Lès États-Unis, à eux seuls, représentent $50 \%$ du marché. Cela n'est pas spécifique à la chirurgie orthopédique et concerne l'ensemble du secteur des biomatériaux. Compte tenu de l'étroitesse du domaine, il n'y a pas ou peu de recherche visant à mettre au point des matériaux spécifiques aux applications biomédicales. De ce fait, la plupart des matériaux dont nous disposons actuellement ont été, d'abord et avant tout, développés pour les grandes aven- 


\section{RÉFÉRENCES}

6. Willert H-G, Buchhorn G, Ungethum $M$. Proposed guideline for the biological testing of orthopaedic implant materials and implants. Biomaterials $1980 ; 1$ : 179-82.

7. Williams DF. Systemic aspects of biocompatibility, vol. 1 et 2 . Boca Raton : CRC Press, 1981

8. Williams DF. Fundamental aspects of biocompatibility, vol. 1 et 2. Boca Raton : CRC Press, 1981.

9. Williams DF. Biocompatibility of clinical implant materials, vol. 1 et 2 . Boca Raton : CRC Press, 1981.

10. Williams DF. Biocompatibility of orthopaedic implants, vol. 1 et 2. Boca Raton : CRC Press, 1982.

11. Heimke G, Griss P, Werner E, Jentschura G. The effects of mechanical factors on biocompatibility tests. J Biomed Eng 1981; 3 : 209-13.

12. Willert HG, Buchhorn G, Buchhorn U, Semlistch M. Tissue response to wear debris in artificial joints. In : Weinstein A, Gibbons D, Brown S, Ruff W, eds. Implant Retrieoal Material and Biological Analysis. NBS special publication 601, US department of commerce, 1981 : 239-67.

13. Allison AC, Harington JS, Birbeck M. An examination of the cytotoxic effects of silica on macrophages. $J$ Exp Med 1966 ; 124 : 141-6.

14. Carter RL, Roe FJC. Induction of sarcomas in rats by solid and fragmented polyethylene, experimental observation and clinical implications. Brit J Cancer 1969; 23 : 401-7.

15. Griss $P$, Werner $E$, Buchinger $R$, Busing CM, Heimke G. Zur Frage der unspezifischen Sarkomentstehung um $\mathrm{Al}_{2} \mathrm{O}_{3}$-Keramische Implantatate. Arch Orthop Traume Surg 1977 ; 90 : 29-36.

16. Jaffray $P$, Adolphe $M$. Cultures cellulaires : intérêt et limites comme technique de remplacement à l'expérimentation animale. Sci Tech Anim Lab 1983 ; 8 : 5-10.

17. Autian J. Toxicological evaluation of biomaterials : Primary acute toxicity screening program. Artif Organs 1977 ; 1: 53-60.

18. Christel P. Potential applications of carbon-carbon composite materials for total joint replacement. In : Perren SM, Schneider E, eds. Biomechanics: Current Interdisciplinary Research. Dordrecht, the Netherlands : Martinus Nijhoff Publ., 1985 ; 61-72.

- Projet de fascicule de documenlation AFNOR, P.S 90-700. Choix des essais permettant d'évaluer la bio574 tures industrielles contemporaines : aérospatiale, énergie nucléaire, etc. C'est le cas des alliages de titane, des matériaux composites, de la céramique d'alumine, pour ne citer que ceux là.

Les biomatériaux à usage orthopédique se présentent sous forme solide et, n'ayant pas d'action pharmacologique, ils échappent à la législation des médicaments et ne sont pas soumis aux procédures d'AMM (autorisation de mise sur le marché). Il s'agit presque toujours de produits de synthèse. Deux grandes catégories de matériaux sont actuellement utilisées : les alliages métalliques et les matières plastiques. Les différentes variétés actuellement utilisées en routine, leurs usages ainsi que leurs qualités et défauts sont rapportés dans le Tableau I. Les matériaux à base de fibres utilisés dans la fabrication des ligaments artificiels ont été volontairement omis du fait d'un recul clinique actuellement insuffisant.

Les alliages métalliques relarguent des ions dont certains ont une toxicité connue (nickel et chrome surtout). Certaines circonstances peuvent favoriser ce relargage : frottement, $\mathrm{pH}$ local. Ce phénomène devient préoccupant avec les implants métalliques poreux destinés à être ancrés directement dans l'os et à être stabilisés par repousse osseuse au sein des porosités. Leur surface développée au contact des tissus est considérable et le relargage ionique ainsi très important. Les métaux ont une rigidité très supérieure à celle de l'os, ils se déforment donc beaucoup moins et perturbent localement les sollicitations mécaniques normalement subies par l'os et nécessaires à sa bonne trophicité. La structure osseuse ainsi modifiée peut être responsable de faillite à long terme des implants par fracture osseuse pathologique ou descellement de l'implant.

Les polymères ont dans l'ensemble des propriétés mécaniques et une stabilité médiocre. Ils s'usent et se déforment. En général, sous forme massive, ils ne posent pas de problème de tolérance tissulaire ; cependant, leurs débris d'usure entraînent une réaction à corps étranger avec prolifération de tissus de granulation inflammatoire. Ceci peut causer des douleurs ou une déstabilisation de l'implant par lyse progressive de l'interface os/implant sous l'action d'enzymes protéolytiques sécrétées par les cellules du granulome.

La céramique d'alumine présente une excellente biocompatibilité à la fois sous forme massive et particulaire. Elle reste cependant relativement peu répandue, car d'utilisation très spécifique, uniquement sous forme de composant de friction pour prothèse totale de hanche. Faute de ne pouvoir disposer de meilleurs matériaux, les alliages métalliques et les polymères du Tableau $I$ restent actuellement les plus utilisés en routine. Historiquement, la plupart de ces matériaux ont été introduits en thérapeutique humaine de façon empirique, sans évaluation expérimentale préalable. Aucun d'entre eux ne s'étant révélé parfaitement adapté à son utilisation, on a vu depuis les années 70 apparaître de nouveaux biomatériaux, candidats potentiels à une utilisation orthopédique. Devant un accroissement exponentiel de ces matériaux et les incertitudes pesant sur leurs performances à long terme chez l'homme, la Food and Drug Administration (FDA) aux Etats-Unis a mis en place une procédure obligeant les promoteurs à faire subir un ensemble d'essais aux biomatériaux, destinés à démontrer leur innocuité et leur efficacité avant leur utilisation chez l'homme [3, 4]. Une législation équivalente a été introduite au Japon, en Angleterre [5], puis en Allemagne [6]. Elle se met en place dans notre pays, grâce aux efforts conjoints de la Commission Nationale d'Homologation du ministère de la Santé et de l'Agence Française de Normalisation (AFNOR)*.

\section{Evaluation préclinique}

L'évaluation des biomatériaux comprend nécessairement et de façon obligatoire la caractérisation physico-chimique (composition, 


\begin{tabular}{|c|c|c|c|c|}
\hline \multicolumn{5}{|c|}{ Tableau I } \\
\hline \multicolumn{5}{|c|}{ PRINCIPAUX MATÉRIAUX UTILISÉS EN ROUTINE CHIRURGICALE ORTHOPÉDIQUE } \\
\hline Matériaux & & $\begin{array}{l}\text { Applications } \\
\text { essentielles }\end{array}$ & Avantages & Inconvénients \\
\hline \multirow{5}{*}{$\begin{array}{l}\text { Alliages } \\
\text { métalliques }\end{array}$} & Acier inoxydable & $\begin{array}{l}\text { Ostéosynthèse : pla- } \\
\text { ques, clous, vis } \\
\text { Prothèses articulaires }\end{array}$ & $\begin{array}{l}\text { Coût peu élevé } \\
\text { Plasticité } \\
\text { Technologie aisée }\end{array}$ & $\begin{array}{l}\text { Corrosion } \\
\text { Fatigue } \\
\text { Rigidité }\end{array}$ \\
\hline & $\begin{array}{l}\text { Chrome } \\
\text { Cobalt coulé }\end{array}$ & $\begin{array}{l}\text { Ostéosynthèse } \\
\text { Prothèses articulaires }\end{array}$ & $\begin{array}{l}\text { Résistance à la } \\
\text { corrosion }\end{array}$ & $\begin{array}{l}\text { Résistance très } \\
\text { médiocre à la fatigue } \\
\text { Pas de plasticité } \\
\text { Relargage de } \mathrm{Cr} \\
\text { Rigidité }\end{array}$ \\
\hline & $\begin{array}{l}\text { Chrome } \\
\text { Cobalt forgé }\end{array}$ & Prothèses articulaires & $\begin{array}{l}\text { Résistance } \\
\text { à la fatigue } \\
\text { et à la corrosion }\end{array}$ & $\begin{array}{l}\text { Technologie difficile } \\
\text { et coût élevé } \\
\text { Rigidité }\end{array}$ \\
\hline & $\begin{array}{l}\text { Nickel } \\
\text { Chrome }\end{array}$ & $\begin{array}{l}\text { Tiges fémorales de } \\
\text { prothèses de hanche }\end{array}$ & $\begin{array}{l}\text { Résistance } \\
\text { à la fatigue } \\
\text { et à la corrosion }\end{array}$ & $\begin{array}{l}\text { Relargage de } \mathrm{Ni} \\
\text { (35\% de Nickel) }\end{array}$ \\
\hline & $\begin{array}{l}\text { Titane } \\
\text { Aluminium } \\
\text { Vanadium }\end{array}$ & Ostéosynthèse & $\begin{array}{l}\text { Grande résistance } \\
\text { à la fatigue } \\
\text { et à la corrosion }\end{array}$ & Coût élevé \\
\hline \multirow{6}{*}{ Polymères } & $\begin{array}{l}\text { Polyéthylène } \\
\text { Haute densité } \\
\text { Haut poids } \\
\text { moléculaire }\end{array}$ & Surfaces articulaires & $\begin{array}{l}\text { Facilité } \\
\text { de fabrication }\end{array}$ & $\begin{array}{l}\text { Usure, fluage, débris } \\
\text { d'usure mal tolérés }\end{array}$ \\
\hline & $\begin{array}{l}\text { Polyéthylène }+ \text { fibres } \\
\text { de carbone }\end{array}$ & Surfaces articulaires & Moins de fluage & Usure \\
\hline & Polyacétal & Surfaces articulaires & $\begin{array}{l}\text { Coût peu élevé } \\
\text { Stérilisable à } \\
\text { l'autoclave }\end{array}$ & $\begin{array}{l}\text { Relargage } \\
\text { de formaldéhyde } \\
\text { Débris d'usure } \\
\text { mal tolérés }\end{array}$ \\
\hline & $\begin{array}{l}\text { Elastomères de } \\
\text { silicone }\end{array}$ & $\begin{array}{l}\text { Prothèses articulaires } \\
\text { Interposition } \\
\text { de glissement }\end{array}$ & Grande élasticité & $\begin{array}{l}\text { Dégradation mécani- } \\
\text { que par absorption de } \\
\text { lipides } \\
\text { Débris mal tolérés }\end{array}$ \\
\hline & $\begin{array}{l}\text { Polymétacrylate } \\
\text { de méthyle }\end{array}$ & $\begin{array}{l}\text { Ancrage des prothè- } \\
\text { ses articulaires } \\
\text { Remplissage } \\
\text { de cavités }\end{array}$ & Usage aisé & $\begin{array}{l}\text { Propriétés mécaniques } \\
\text { médiocres } \\
\text { Toxicité générale } \\
\text { Température de } \\
\text { polymérisation trop } \\
\text { élevée } \\
\text { Vieillissement }\end{array}$ \\
\hline & $\begin{array}{l}\text { Polyéthylène }+ \text { fibres } \\
\text { de carbone }\end{array}$ & Surfaces articulaires & Moins de fluage & Usure \\
\hline Céramiques & Alumine frittée & Surfaces articulaires & $\begin{array}{l}\text { Biocompatibilité } \\
\text { Friction très basse }\end{array}$ & Technologie difficile \\
\hline
\end{tabular}


densité, microstructure, propriétés thermodynamiques, électriques, etc.), mécanique statique et dynamique (élasticité, résistance, dureté, fatigue, etc.), le passage sur banc d'essai (usure, friction, corrosion, vieillissement, etc.) et enfin l'investigation des performances biologiques du matériau. Ce n'est qu'après avoir franchi avec succès toutes ces étapes que le biomatériau pourra faire l'objet d'une utilisation clinique, d'abord limitée dans le cadre d'un essai thérapeutiquè, puis généralisée.

Pour démontrer l'innocuité et l'efficacité d'un biomatériau, il est nécessaire, sur le plan de l'évaluation biologique, de lui faire subir des essais de biocompatibilité et de biofonctionnalité. Ces derniers tests concernent en fait le produit final (le plus souvent un implant) qui peut lui-même être constitué de plusieurs biomatériaux.

Le choix des essais de biocompatibilité est basé sur la connaissance des facteurs toxicologiques qui la contrôlent $[7,8,9,10]$.

Les facteurs chimiques. Le relargage ionique dans les tissus avoisinants, en provenance des alliages métalliques, est généralement en rapport avec leurs éléments constitutifs majoritaires (bien qu'il puisse survenir un relargage préférentiel en provenance de certaines phases plus sensibles que d'autres à la corrosion). Il n'en va pas de même des polymères. Leur toxicologie est en rapport avec la petite fraction soluble de monomères résiduels et d'additifs. La toxicologie de ces derniers peut être différente de celle de l'additif d'origine qui peut se transformer par dégradation enzymatique lors de son passage dans les tissus.

Les facteurs électriques. Les courants de polarisation anodique ou cathodique que peuvent présenter les métaux, quand ils sont utilisés sous forme d'électrodes intramus: culaire ou intraosseuse par exemple, conditionnent considérablement leur corrosion. La polarisation anodique entraîne une dissolution accrue des ions métalliques dans l'organisme.

Les propriétés de surface des 576 matériaux. Les alliages ont la pro- priété de se recouvrir spontanément d'une couche d'oxyde métallique constituant un film de passivation protecteur, diminuant les phénomènes de relargage ionique. On peut en augmenter artificiellement l'épaisseur par des processus électrochimiques et le rendre ainsi plus résistant. La nature hydrophile ou hydrophobe de la surface d'un matériau dépend de sa tension superficielle. Elle conditionne la mouillabilité de surface du matériau et l'aptitude des protéines à s'adsorber. Les matériaux les plus hydrophiles sont généralement les mieux tolérés.

Les interactions mécaniques tissu/matériau. La nature des contraintes mécaniques sollicitant l'interface tissu/matériau (traction, compression ou cisaillement) conditionne la réponse tissulaire. Dans le cas d'un implant intra-osseux, cette interface sera le siège d'une couche de tissu fibreux négligeable ou absente au niveau des zones sollicitées en compression, tandis qu'une couche de tissu fibreux se développera au niveau des interfaces en traction. Dans le cas le plus général, l'interface os/implant est soumis essentiellement à des contraintes combinées de flexion/torsion ; celles-ci induisent des contraintes de cisaillement à l'interface dont l'intensité sera d'autant plus grande que les différences de rigidité entre le tissu osseux et le matériau seront importantes [11].

Les facteurs géométriques. La réponse cellulaire et tissulaire à un matériau, à court et moyen terme, diffère selon que celui-ci se présente sous forme massive ou particulaire. La réaction tissulaire induite par des particules sera toujours plus importante que celle observée pour le matériau massif. Elle dépend de la granulométrie, de la forme géométrique et de la quantité de particules présentes $[12,13]$. L'irritation tissulaire chronique peut quant à elle aboutir à long terme à la production de tumeurs sarcomateuses. Tous les matériaux, même les mieux tolérés, tel l'alumine ou le polyéthylène, sont susceptibles d'induire des tumeurs malignes chez l'animal $[14,15]$. Les matériaux sous forme massive sont plus cancérigènes que sous forme particulaire. Les rapports surface/volume jouent un rôle critique dans ces phénomènes.

\section{Choix des essais de biocompatibilité}

La liste des essais disponibles est indiquée dans le Tableau II. Cette liste est loin d'être exhaustive et des essais évaluant d'autres aspects de la biocompatibilité sont en cours de standardisation, comme la tolérance aux débris d'usure. Les tests in vitro ont l'avantage de donner des réponses plus rapides avec un coût moins élevé que les tests in vivo [16]. Le choix de la batterie des essais dépend de la durée de contact du matériau avec les tissus [17]. Le Tableau II indique les procédures d'essai de biocompatibilité à effectuer en fonction de l'application potentielle du matériau.

La biofonctionnalité est un ensemble de tests conçus pour évaluer et vérifier le bon fonctionnement du biomatériau in vivo, en condition de service. Par exemple : vérifier qu'une prothèse articulaire à revêtement poreux est réellement colonisée par du tissu osseux. Ces expériences fonctionnelles doivent être réalisées sur gros animaux de façon à ce que l'environnement biomécanique, la dimension des implants et les processus métaboliques se rapprochent le plus possible de ceux rencontrés chez l'homme. Chaque protocole est ainsi adapté au but recherché. L'évaluation de la biofonctionnalité d'un matériau constitue l'étape ultime avant d'effectuer l'essai clinique.

\section{Ne pas freiner I'innovation}

Progressivement, un système structuré d'évaluation et d'homologation des biomatériaux se met en place. Il permet évidemment de mieux protéger les patients contre les dangers éventuels liés à l'utilisation de matériaux au devenir et aux performances in vivo inconnus. Il doit cependant con- 
server une certaine souplesse pour ne pas freiner la créativité des chercheurs et des industriels. A ce titre, la situation des États-Unis constitue un exemple à méditer : la FDA a fait adopter par le congrès, en 1976, un amendement mettant en place une législation rigoureuse et contraignante concernant l'autorisation de commercialisation des matériaux et systèmes médicaux. Pour la chirurgie orthopédique, cette législation oblige à faire subir à tout nouveau produit un ensemble d'essais de laboratoire sur des animaux, lourds et coûteux, avant de passer à un essai clinique contrôlé uni puis pluricentrique. Ce n'est qu'après avoir franchi toutes ces étapes que la commercialisation du produit est éventuellement accordée. Cette autorisation n'est pas automatique, même si tous les essais ont été effectués avec succès. L'interprétation de ceux-ci est sujette à des variations, dépendan- tes des experts constituant le comité examinant les dossiers. Ainsi, si la FDA constitue une protection réelle pour les patients, elle a pour effet secondaire de freiner considérablement l'innovation et le progrès médical. Si perfectionnés que soient les protocoles utilisés, il persiste toujours une inconnue dans l'extrapolation des résultats obtenus chez l'animal à la clinique humaine. La mise au point de nouvelles méthodes d'évaluation peut diminuer cette inconnue, mais la responsabilité du médecin restera toujours l'élément déterminant dans la décision de l'essai clinique.

Le coût total de l'évaluation d'un nouveau biomatériau orthopédique se chiffre par millions de francs. Sa durée varie entre cinq et dix ans. Pour ces raisons, seules les grandes entreprises possédant des départements de recherche et de développement peuvent apporter des innovations importantes. On
Tableau II

PROCÉDURES D'ESSAIS DE BIOCOMPATIBILITÉ À APPLIQUER A UN BIOMATÉRIAU POTENTIEL EN FONCTION DE LA DURÉE DE CONTACT BIOMATÉRIAU / TISSU OSSEUX (selon AFNOR Pr S90-700)

\begin{tabular}{|l|c|c|c|}
\hline Essais & $\begin{array}{c}\text { contact de moins } \\
\text { de 6 heures }\end{array}$ & $\begin{array}{c}\text { contact de } \\
6 \text { heures à 30 j. }\end{array}$ & $\begin{array}{c}\text { contact plus de } \\
30 \mathrm{j} .\end{array}$ \\
\hline Cytotoxicité & - & $\times$ & $\times$ \\
Pouvoir mutagène & - & $\times$ & $\times$ \\
Toxicité systémique & - & $\times$ & $\times$ \\
$\begin{array}{l}\text { Injection } \\
\text { intradermique }\end{array}$ & $\times$ & $\times$ & $\times$ \\
Sensibilisation & $\times$ & $\times$ & $\times$ \\
$\begin{array}{l}\text { Implantation à court } \\
\text { terme }\end{array}$ & - & $\times$ & - \\
$\begin{array}{l}\text { Implantation à long } \\
\text { terme }\end{array}$ & - & - & $\times$ \\
Pouvoir carcinogène & - & $\times$ & $\times$ \\
\hline Pyrogènes & $\times$ & & \\
\hline
\end{tabular}

comprend pourquoi les PME sont mal placées dans la course aux nouveaux biomatériaux.

\section{Le futur}

Celui-ci passe par une meilleure connaissance du tissu squelettique, la mise au point de nouvelles méthodes d'évaluation et enfin l'utilisation de nouveaux matériaux mieux adaptés au cahier des charges.

Une meilleure connaissance du tissu squelettique. Il s'agit surtout de préciser les lois du remodelage osseux en fonction des sollicitations mécaniques et d'établir la relation quantitative existant entre contraintes et ostéogenèse. La connaissance de cette relation doit permettre d'optimiser les caractéristiques mécaniques des implants de façon à perturber le moins possible la distribution et l'amplitude des contraintes physiologiques agissant normalement sur le tissu osseux.

Des méthodes d'évaluation quantitatives. Nous ne disposons à ce jour d'aucun critère quantitatif représentatif en valeur absolue de la biocompatibilité d'un matériau. Actuellement, les évaluations se font, toujours de façon comparative avec un matériau de référence ayant un passé clinique ancien. Elles consistent surtout à observer la réaction cellulaire ou tissulaire au contact du matériau ou de ses produits de relargage en quantifiant nombre et types de cellules, métabolites relargués ou consommés par celles-ci.

Des nouveaux biomatériaux. Un certain nombre de matériaux ont un avenir potentiel à plus ou moins court terme. Nous citerons les principaux :

- Les matériaux composites qui sont constitués d'une matrice dans laquelle sont réparties des fibres de longueur et d'orientation précises, sont intéressants à plus d'un titre. Ils ont été conçus essentiellement pour des applications aérospatiales et leurs caractéristiques mécaniques se rapprochent considérablement de celles du tissu osseux (figure $1 \mathrm{~A}$ et $B$ p. 578). Les matériaux composites qui font actuellement l'objet d'essais cliniques sont essentielle- 


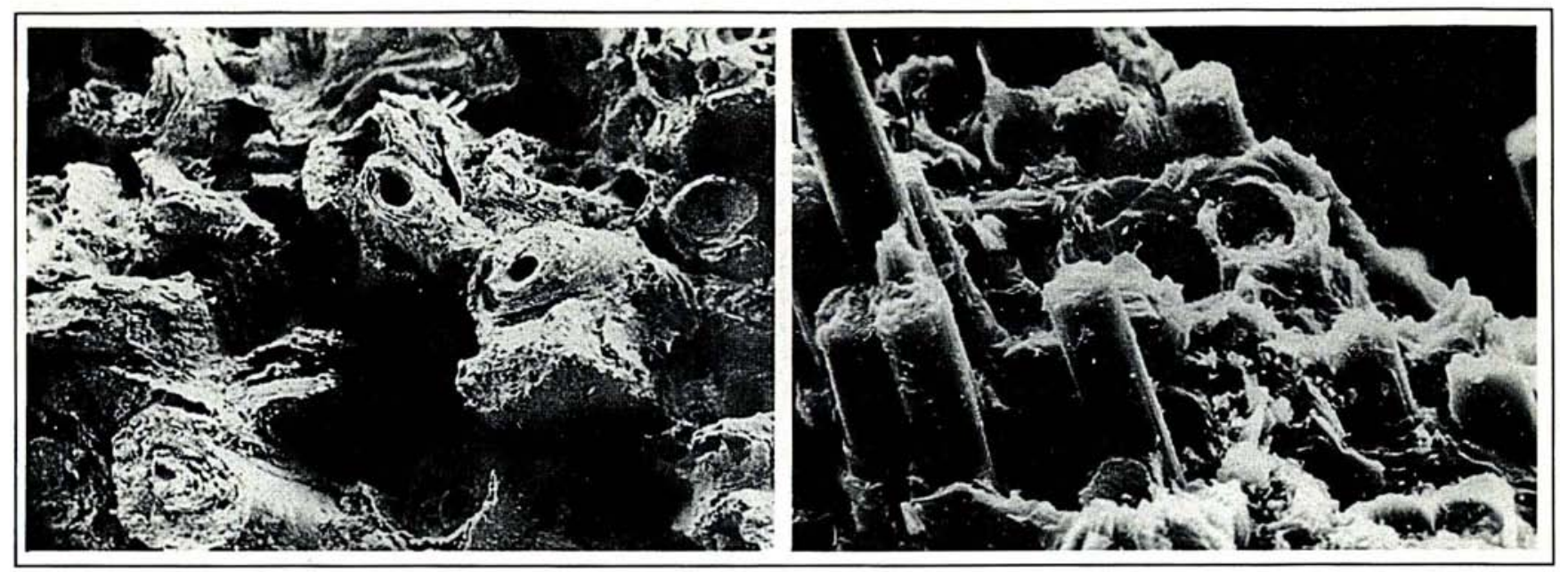

Figure 1. Aspect de la surface en microscopie à balayage après fracture de l'os cortical et d'un matériau composite. Noter la similitude de structure entre les deux matériaux. A gauche : Os cortical humain. A droite : Matériau composite carbone-carbone.

ment des matrices de polysulfone renforcées par fibres de carbone ou des matrices de carbone pyrolitique, elles-mêmes renforcées par des fibres de carbone [18]. D'autres matériaux composites sont à l'étude ; tous sont essayés en tant qu'implant d'ostéosynthèse ou d'élément fémoral de prothèse totale de hanche. Dans l'ensemble, leur résistance mécanique n'est pas aussi élevée que celle des alliages métalliques, mais leur grande élasticité perturbe moins le remodelage du tissu osseux que les implants métalliques équivalents.

- Les céramiques à base de phosphate de calcium (apatites et dérivés) ou de carbonate de calcium (calcites, coraux et dérivés) ont un grand avenir en tant que matériaux substitutifs du tissu osseux. Selon leur structure cristalline, ils sont résorbables ou non. Ils sont " bioactifs " car ils échangent des ions avec le tissu osseux du voisinage et dans tous les cas ils présentent des propriétés ostéogéniques très intéressantes. Leurs propriétés mécaniques varient selon la nature chimique du matériau et sa porosité. Ils sont surtout destinés à être utilisés comme matériaux de comblement des cavités osseuses et appelés à remplacer les greffes cortico-spongieuses. Simultané578 en stomatologie. Ils peuvent aussi être utilisés sous forme de matériaux composites au sein d'une matrice collagène qui stimule l'activité cellulaire.

Devant les insuffisances des matériaux actuels, il est bien évidemment nécessaire d'en développer de nouveaux, mieux adaptés au cahier des charges des implants orthopédiques. Ceci implique des recherches longues et coûteuses nécessitant d'importants investissements. La question essentielle est de savoir si, avec un biomatériau nouveau, candidat à l'utilisation orthopédique, on peut espérer obtenir à long terme de meilleurs résultats que ceux observés avec les matériaux actuellement utilisés. Seule l'expérimentation clinique et l'épreuve du temps apportent la réponse à cette question. Le bien fondé de l'utilisation d'un nouveau matériau ou la justification d'un concept nouveau ne peuvent donc trouver leur démonstration qu'à posteriori. Il faut être conscient que l'amélioration de la qualité des résultats des interventions orthopédiques tient d'abord à la sélection des indications et à l'utilisation d'une bonne technique chirurgicale. Le matériau ou le dessin de l'implant ne peuvent ajouter au succès que si les éléments précédents sont respectés

\section{Summary}

The introduction of new materials in clinical practice needs large investments and longlasting research. The specifications of present and potential biomaterials are critically reviewed in term of biocompatibility, biofunctionality, cost and marketability. Besides the in vitro and animal evaluations, clinical trials and long-term follow-up are mandatory to provide a definite answer to the question of biomaterials performances in patients. New biomaterials and implant design will improve the quality of the clinical results only if surgery is correctly performed.

TIRÉS A PART

P. Christel : laboratoire de recherches orthopédiques, U.A. Cnrs 1161, 10, avenue de Verdun, 75010 Paris. 